\title{
Malignant Orbit Hemangiopericytoma
}

National Cancer Institute

\section{Source}

National Cancer Institute. Malignant Orbit Hemangiopericytoma. NCI Thesaurus. Code C4544.

A malignant hemangiopericytoma arising in the orbit. 